\title{
Comparison of oxygenation and ventilation parameters between proseal laryngeal mask airway and endotracheal tube in patients undergoing laparoscopic surgeries: a prospective observational study
}

\author{
Deepak Devendran ${ }^{1}$, Karthik Jain $\mathbf{M}^{2, *}$, Vasudeva Upadhyaya K.S ${ }^{3}$ \\ ${ }^{1}$ Senior Resident, ${ }^{\mathbf{2}}$ Associate Professor, ${ }^{3}$ Professor, Dept. of Anaesthesiology, St. John's Medical College, Bangalore, Karnataka, \\ India
}

*Corresponding Author: Karthik Jain M

Email: karthikjain@yahoo.com

Received: $17^{\text {th }}$ May, 2018

Accepted: $26^{\text {th }}$ September, 2018

\begin{abstract}
Introduction: Use of endotracheal tube (ETT) has many limitations. There is a need for an airway device with similar safety and good side effect profile. In this prospective observational study, the oxygenation and ventilation parameters between patients who were inserted Proseal laryngeal mask airway (PLMA) or ETT for laparoscopic surgeries were compared.

Materials and Methods: After ethical committee approval, informed consent was obtained from sixty patients (thirty in each group) posted for elective laparoscopic surgery. Following induction of general anaesthesia and paralysis, PLMA or ETT was inserted and pressure control mode was used for ventilation. The following parameters were measured: a. Oxygen saturation and changes in $\mathrm{FiO}_{2}$ needed to ensure a target $\mathrm{SpO}_{2}>95 \%, \mathrm{EtCO}_{2}$ and changes in respiratory rate and inspiratory pressure needed to ensure a target $\mathrm{EtCO}_{2}$ between $35-45 \mathrm{mmHg}$. b. Insertion characteristics of airway device and gastric tube, hemodynamic responses, incidence of complications. Statistical Analysis: Chi-square test was used to analyze qualitative data. Independent $t$ test was used to assess quantitative variables. Repeated measures ANOVA was used to compare the outcome measures over time adjusted for duration of surgery and anaesthesia. $P$ values $<0.05$ considered significant.

Results: Both groups were comparable with respect to age, gender and weight. Oxygenation and ventilation parameters achieved with both ETT and PLMA were comparable and satisfactory. Haemodynamic parameters increased significantly in ETT group compared to PLMA group during airway insertion and removal. Also, postoperative sore throat was comparatively less in the PLMA group.

Conclusion: Hence we conclude that PLMA may be considered as an alternative to ETT in airway management of patients undergoing laparoscopic surgeries with comparable oxygenation and ventilation parameters and improved side effect profile.
\end{abstract}

Keywords: Endotracheal tube, Proseal laryngeal mask airway, Laryngoscopy, Pneumoperitoneum.

\section{Introduction}

Use of endotracheal tube (ETT) has been a standard practice to maintain patient's airway during general anaesthesia. ${ }^{1}$ Supraglottic airway devices (SAD) with continuing improvement in characteristics are evolving and are replacing or used as a safe alternative to ETT in several clinical settings. The advantages of endotracheal intubation include reliability and minimal risk of aspiration. Direct laryngoscopy for intubation necessitates training and practice to attain good skill. Complications of laryngoscopy and intubation include hypertensive episodes, myocardial injury, arrhythmias etc. because of autonomic stimulation. ${ }^{2}$ Supraglottic airway devices can avoid such complications. ${ }^{3}$

Among various supraglottic devices, Proseal laryngeal mask airway (PLMA) has many advantages including better airway seal because of a dorsal cuff and provision of drainage of regurgitated gastric fluid via the drain tube. PLMA can be used for both spontaneous and controlled ventilation. It permits higher airway pressures compared to other supraglottic devices which may deem it better suited for laparoscopic surgeries. There are limited studies in India comparing the working performance of PLMA and ETT in laparoscopic surgeries. Therefore we planned to conduct a study comparing working characteristics of PLMA and ETT in these surgeries.

The primary aim of the study was to compare parameters for oxygenation and ventilation achieved with PLMA as compared to the ETT. The parameters compared were: $1 . \mathrm{FiO}_{2}$ changes to maintain $\mathrm{SpO}_{2}>$ $95 \%$. 2. Changes in inspiratory pressure and respiratory rate to maintain an end-tidal carbon dioxide $\left(\mathrm{EtCO}_{2}\right)$ between 35-45 mmHg. The secondary objectives included: a. Changes in heart rate and blood pressure accompanying insertion/removal of PLMA as compared to ETT. b. Time taken to achieve an effective airway. c. Time taken for insertion of Ryles's tube as well as ease of insertion. d. Incidence of complications between the two groups (sore throat, airway trauma). e. Airway interventions required if any and cuff leak pressures.

\section{Materials and Methods}

Based on power analysis on the study done by Namita $\mathrm{S}$ et $\mathrm{al},{ }^{4}$ the sample size required to observe a significant difference in study parameters was 30 per group. The sample size was computed assuming a twosided test with Type 1 error of $5(\alpha=0.05)$ and power of 
0.8. An observational study of prospective type was planned. After ethics committee approval, sixty patients aged between 18 to 60 years, American Society of Anaesthesiology (ASA) class I and II posted for laparoscopic surgeries under general anaesthesia were chosen for the study. The study subjects were distributed equally into two groups.

Group P $(\mathrm{n}=30)$ - Patients receiving PLMA of size 3, 4, or 5 (according to weight)

Group ETT $(n=30)$ - Patients receiving ETT (size 7.5 for females and 8.5 for males)

Exclusion criteria included patients with anticipated difficult airway including morbid obesity $\left(\mathrm{BMI}>35 \mathrm{~kg} / \mathrm{m}^{2}\right)$ and cervical spine pathology, cardiopulmonary illness, increased risk of aspiration (GERD, hiatus hernia, obstetric patients, and inadequate fasting status), history of recent respiratory tract infection and patient refusal.

Written informed consent was taken from all patients satisfying the inclusion and exclusion criteria. According to the type of the airway device they received, ETT vs. PLMA (which was decided by the concerned consultant anesthetist), they were allocated to the respective group and were observed for the required parameters.

The anesthetic procedure followed was as per standard department protocol, which is as follows: All patients were kept fasting overnight and received Tablet Alprazolam $0.25 \mathrm{mg}$ and Capsule Omeprazole $20 \mathrm{mg}$, orally, night before surgery. Routine monitoring including $\mathrm{SpO}_{2}, \mathrm{ECG}, \mathrm{NIBP}, \mathrm{EtCO}_{2}$ and Temperature was initiated in all patients in the operation theatre. Premedication with $0.004 \mathrm{mg} / \mathrm{kg}$ of Glycopyrrolate, $0.02 \mathrm{mg} / \mathrm{kg}$ of Midazolam and $0.08 \mathrm{mg} / \mathrm{kg}$ of Ondansetron intravenously (i.v.) was administered by the anaesthetic team prior to the induction of general anaesthesia. General anaesthesia was induced with fentanyl 2 microgram $/ \mathrm{kg}$ and propofol $1 \% \quad 1-2 \mathrm{mg} / \mathrm{kg}$ i.v. after pre-oxygenation for 3 minutes with $100 \%$ oxygen. Neuromuscular blockade was achieved with atracurium $0.5 \mathrm{mg} / \mathrm{kg}$ i.v.

Following induction and paralysis, the corresponding airway was inserted in each group as per standard recommendation, by the anaesthetist. Proper insertion of airway device was confirmed by bilateral equal air entry over chest, square wave capnography, absence of audible leak with peak airway pressure (PAP) of $20 \mathrm{~cm} \mathrm{H} \mathrm{H}_{2} \mathrm{O}$, and absence of gastric insufflations by auscultation over epigastrium. General anaesthesia was maintained with air, oxygen and isoflurane with pressure controlled ventilation and divided doses of atracurium and fentanyl. The ventilator settings were kept initially at $\mathrm{FiO}_{2}$ of 0.40 , respiratory rate of 12, and inspiratory pressure of $15 \mathrm{cms}_{2} \mathrm{O}$, and $\mathrm{I} / \mathrm{E}$ ratio of $1: 2$. $\mathrm{FiO}_{2}$, respiratory rate and inspiratory pressure was varied to maintain a target $\mathrm{SpO}_{2}>95 \%$ and $\mathrm{ETCO}_{2}$ between $35-45 \mathrm{mmHg}$. After completion of surgery, $\mathrm{FiO}_{2}$ was increased to $100 \%$ and neuromuscular blockade was reversed with Neostigmine 50 microgram $/ \mathrm{kg}$ and. Glycopyrrolate $0.01 \mathrm{mg} / \mathrm{kg}$ i.v and airway device (PLMA or ETT) was removed after adequate clinical recovery of neuromuscular block and return of consciousness.

Perioperatively the following outcomes were measured from baseline values till 5 minutes after removal of the airway:

\section{Primary Objectives}

1. $\mathrm{FiO}_{2}$ changes to maintain $\mathrm{SpO}_{2}>95 \%$.

2. Changes in inspiratory pressure and respiratory rate to maintain an end-tidal carbon dioxide $\left(\mathrm{EtCO}_{2}\right)$ between $35-45 \mathrm{mmHg}$

The secondary objectives

a. Changes in heart rate and blood pressure accompanying insertion/removal of PLMA as compared to ETT.

b. Time taken to achieve an effective airway.

c. Time taken for insertion of Ryle's tube as well as ease of insertion.

d. Incidence of complications between the two groups (sore throat, airway trauma).

e. Airway interventions required if any and cuff leak pressures. (Oropharyngeal leak pressure (OPLP) was determined by closing the expiratory valve against a fixed gas flow of $5 \mathrm{l} / \mathrm{min}$ and recording the airway pressure at which equilibrium was reached. The airway pressure was not allowed to exceed $40 \mathrm{~cm} \mathrm{H}_{2} \mathrm{O}$ )

\section{Statistical Analysis}

Data was entered into Microsoft Excel data sheet and was analyzed using SPSS 22 version software. Categorical data was represented in the form of frequencies and proportions. Chi-square test was used as test of significance for qualitative data. Continuous data was represented as mean and SD. Independent t test was used as test of significance to identify the mean difference between two quantitative variables. Repeated measures ANOVA was used to compare the outcome measures over time adjusted for duration of surgery and anaesthesia. As per standard statistical models, $\mathrm{P}$ values $<0.001$ were considered very highly significant, $<0.01$ highly significant, $<0.05$ significant.

\section{Observations and Results}

Average age, weight, height, BMI as well as gender and ASA class distribution between groups was similar (Table 1). Duration of pneumoperitoneum between groups was also similar. But difference in mean duration of surgery and anaesthesia between two groups was significant (Table 2).

Although there was no significant difference in mean time taken for airway device insertion between two groups, difference in mean RT insertion time between two groups was statistically significant (Table 3). In Group $\mathrm{P}$, airway device was inserted in $1^{\text {st }}$ 
attempt among $76.7 \%, 2^{\text {nd }}$ attempt in $13.3 \%$ and $3^{\text {rd }}$ attempt in $10 \%$ of patients. In Group ETT, airway device was inserted in $1^{\text {st }}$ attempt in $100 \%$ of subjects. In Group P, airway insertion was easy, moderately easy and difficult in 80, 10 and 10 percent of patients respectively. In ETT group, airway insertion was easy in $100 \%$. This difference in number of attempts and ease of insertion for airway device between two groups was statistically significant (Table 4). The difference in number of attempts and ease of insertion for Ryle's tube between two groups was statistically not significant but the difference in mean Ryle's tube insertion time was significant. Mean time for Ryle's tube insertion was $21.2 \pm 12.3$ seconds in group $\mathrm{P}$ and $74.8 \pm 62.5$ seconds in group ETT (Table 5).

In Group P, no patient had PONV, $10 \%$ had sore throat and $20 \%$ had blood staining. In Group ETT, $6.7 \%$ had PONV, 33.3\% had sore throat and $13.3 \%$ had blood staining. There was significant difference in Sore throat between two groups (Fig. 1).

Mean Intra-abdominal pressure (IAP) in Group P was $13 \pm 1.5 \mathrm{mmHg}$ and in Group ETT was $12.4 \pm 0.7$ $\mathrm{mmHg}$. This difference in mean IAP between two groups was not statistically significant. Mean OPLP in Group $\mathrm{P}$ was $31 \pm 1.6 \mathrm{~cm} \mathrm{H}_{2} \mathrm{O}$.

Mean HR was significantly higher in Group ETT compared to Group P at Airway insertion, 1 min to 3 min after airway insertion, at airway removal and till 5 min after airway removal (Fig. 2). Mean MAP was significantly high in Group ETT compared to Group P at Airway insertion, $1 \mathrm{~min}$ to $3 \mathrm{~min}$ after airway insertion, $10 \mathrm{~min}$ after insufflations, at airway removal and 1 min after airway removal (Fig. 3).
$\mathrm{FiO}_{2}$ changes to maintain $\mathrm{SPO}_{2}>95 \%$ was not needed in both groups at all the intervals of follow-up. $\mathrm{FiO}_{2}$ pattern in both the groups was similar at all intervals (Fig. 4). Although $\mathrm{SPO}_{2}$ was maintained above target level of $95 \%$ without changes in $\mathrm{FiO}_{2}$ settings, Mean $\mathrm{SpO}_{2}$ was significantly high in Group $\mathrm{P}$ compared to Group ETT at airway insertion $(\mathrm{p}=0.04), 3$ min after airway insertion $(\mathrm{p}<0.001), 15 \mathrm{~min}$ after Insufflation $(p=0.03)$, at Desufflation $(p<0.001)$ and airway removal $(\mathrm{p}=0.03)$ (Fig. 5)

Changes in respiratory rate and inspiratory pressure was needed in both groups to maintain $\mathrm{EtCO}_{2}$ within target values of $35-45 \mathrm{mmHg}$. There was significant difference in mean $\mathrm{ETCO}_{2}$ between two groups at airway insertion $(\mathrm{p}=0.02), 3 \mathrm{~min}$ after insertion $(\mathrm{p}=0.001)$, from $10 \mathrm{~min}$ to $70 \mathrm{~min}$ after Insufflation $(p<0.001)$ and again at Desufflation $(p=0.003)$. At these initial intervals mean $\mathrm{ETCO}_{2}$ was significantly high in Group P than in Group ETT. After Insufflation mean $\mathrm{ETCO}_{2}$ was significantly high in Group ETT than in Group P (Fig. 6). Mean respiratory rate was significantly high in Group $\mathrm{P}$ than in Group ETT from $10 \mathrm{~min}$ to $40 \mathrm{~min}$ after Insufflation $(\mathrm{p}<0.05)$ and again at Desufflation $(\mathrm{p}=0.003), 3 \mathrm{~min}$ and $5 \mathrm{~min}$ after airway removal $(p<0.05$ ) (Fig. 7). Mean inspiratory pressure was significantly high in Group $P$ than in Group ETT from $10 \mathrm{~min}$ to $45 \mathrm{~min}(\mathrm{p}<0.001), 60 \mathrm{~min}$ $(\mathrm{p}=0.03), 70 \mathrm{~min}(\mathrm{p}=0.02)$ and $75 \mathrm{~min}(\mathrm{p}=0.03)$ after Insufflation and again at Desufflation ( $\mathrm{p}=0.01$ ) (Fig. 8). Changes in Mean TV was secondary to changes in inspiratory pressure and respiratory rate and was significantly high in Group ETT than in Group P, from 3 min after airway insertion till $15 \mathrm{~min}$ after Insufflation ( $\mathrm{p}=0.01)$ (Fig. 9).

Table 1: Demographic profile of subjects between two groups

\begin{tabular}{|l|c|c|c|c|}
\hline \multicolumn{2}{|c|}{} & \multicolumn{2}{|c|}{ Group } & \multirow{2}{*}{ P value } \\
\cline { 3 - 4 } \multicolumn{2}{|c|}{} & Group P & Group ETT & \\
\hline Age (years) & Mean \pm SD & $42.6 \pm 13.1$ & $43.6 \pm 14.8$ & 0.783 \\
\hline Weight (kilograms) & Mean \pm SD & $65.6 \pm 13.5$ & $67.1 \pm 10.1$ & 0.628 \\
\hline Height (meters) & Mean \pm SD & $1.6 \pm 0.1$ & $1.6 \pm 0.1$ & 0.399 \\
\hline BMI (kilogram/meter ${ }^{2}$ ) & Mean \pm SD & $24.9 \pm 4.1$ & $25.1 \pm 2.9$ & 0.816 \\
\hline
\end{tabular}

Table 2: Duration in minutes of Pneumoperitoneum, Surgery and anaesthesia comparison between two groups

\begin{tabular}{|l|c|c|c|c|}
\hline \multicolumn{2}{|l|}{} & \multicolumn{2}{|c|}{ Group } & \multirow{2}{*}{ P value } \\
\cline { 3 - 5 } \multicolumn{2}{|l|}{} & Group P & Group ETT & \\
\hline Duration of Pneumoperitoneum (mins) & Mean \pm SD & $47.7 \pm 19.4$ & $61.8 \pm 35.1$ & 0.058 \\
\hline Duration of Surgery (mins) & Mean \pm SD & $66.7 \pm 26.5$ & $86.7 \pm 35.9$ & 0.017 \\
\hline Duration of Anaesthesia (mins) & Mean \pm SD & $93.8 \pm 27.0$ & $120.3 \pm 38.2$ & 0.003 \\
\hline
\end{tabular}

Table 3: Time taken for airway device insertion and Ryle`s tube insertion time comparison between two groups

\begin{tabular}{|l|c|c|c|c|}
\hline \multicolumn{2}{|c|}{} & \multicolumn{2}{c|}{ Group } & \multirow{2}{*}{ P value } \\
\cline { 3 - 5 } \multicolumn{2}{|c|}{} & Group P & Group ETT & \\
\hline Time taken for airway device insertion $(\mathrm{sec})$ & Mean \pm SD & $30.6 \pm 35.1$ & $32.2 \pm 11.0$ & 0.816 \\
\hline RT insertion Time $(\mathrm{sec})$ & Mean \pm SD & $21.2 \pm 12.3$ & $74.8 \pm 62.5$ & $<0.001$ \\
\hline
\end{tabular}


Table 4: Airway device insertion characteristics - Number of attempts and ease of insertion comparison between two groups

\begin{tabular}{|l|c|c|c|c|c|c|}
\hline Airway device & \multicolumn{5}{|c|}{ Group } & \multirow{2}{*}{ P value } \\
\cline { 3 - 6 } \multicolumn{2}{|c|}{} & \multicolumn{2}{|c|}{ Group P } & \multicolumn{2}{c|}{ Group ETT } & \\
\cline { 3 - 6 } & Count & $\mathbf{\%}$ & Count & $\mathbf{\%}$ & \\
\hline \multirow{3}{*}{ Number of Attempts } & 1 & 23 & $76.7 \%$ & 30 & $100.0 \%$ & \multirow{2}{*}{0.019} \\
\cline { 2 - 6 } & 2 & 4 & $13.3 \%$ & 0 & $0.0 \%$ & \\
\cline { 2 - 6 } & 3 & 3 & $10.0 \%$ & 0 & $0.0 \%$ & \\
\hline \multirow{3}{*}{ Ease of Insertion } & Easy & 24 & $80.0 \%$ & 30 & $100.0 \%$ & \multirow{2}{*}{0.036} \\
\cline { 2 - 6 } & Moderate & 3 & $10.0 \%$ & 0 & $0.0 \%$ & \\
\cline { 2 - 6 } & Difficult & 3 & $10.0 \%$ & 0 & $0.0 \%$ & \\
\hline
\end{tabular}

Table 5: Gastric Tube insertion, number of attempts and ease of insertion comparison between two groups

\begin{tabular}{|l|c|c|c|c|c|c|}
\hline \multicolumn{2}{|c|}{ Gastric Tube } & \multicolumn{4}{c|}{ Group } & \multirow{2}{*}{ P value } \\
\cline { 3 - 6 } \multicolumn{2}{|c|}{} & \multicolumn{2}{|c|}{ Group P } & Group ETT & \\
\cline { 2 - 6 } & Count & \% & Count & \% & \\
\hline \multirow{3}{*}{ No of Attempts } & 1 & 27 & $90.0 \%$ & 21 & $70.0 \%$ & \multirow{2}{*}{0.093} \\
\cline { 2 - 6 } & 2 & 3 & $10.0 \%$ & 6 & $20.0 \%$ & \\
\cline { 2 - 6 } & 3 & 0 & $0.0 \%$ & 3 & $10.0 \%$ & \\
\hline \multirow{3}{*}{ Ease of Insertion } & Grade 1 & 25 & $83.3 \%$ & 23 & $76.7 \%$ & \multirow{2}{*}{0.202} \\
\cline { 2 - 6 } & Grade 2 & 5 & $16.7 \%$ & 4 & $13.3 \%$ & \\
\cline { 2 - 6 } & Grade 3 & 0 & $0.0 \%$ & 3 & $10.0 \%$ & \\
\hline
\end{tabular}

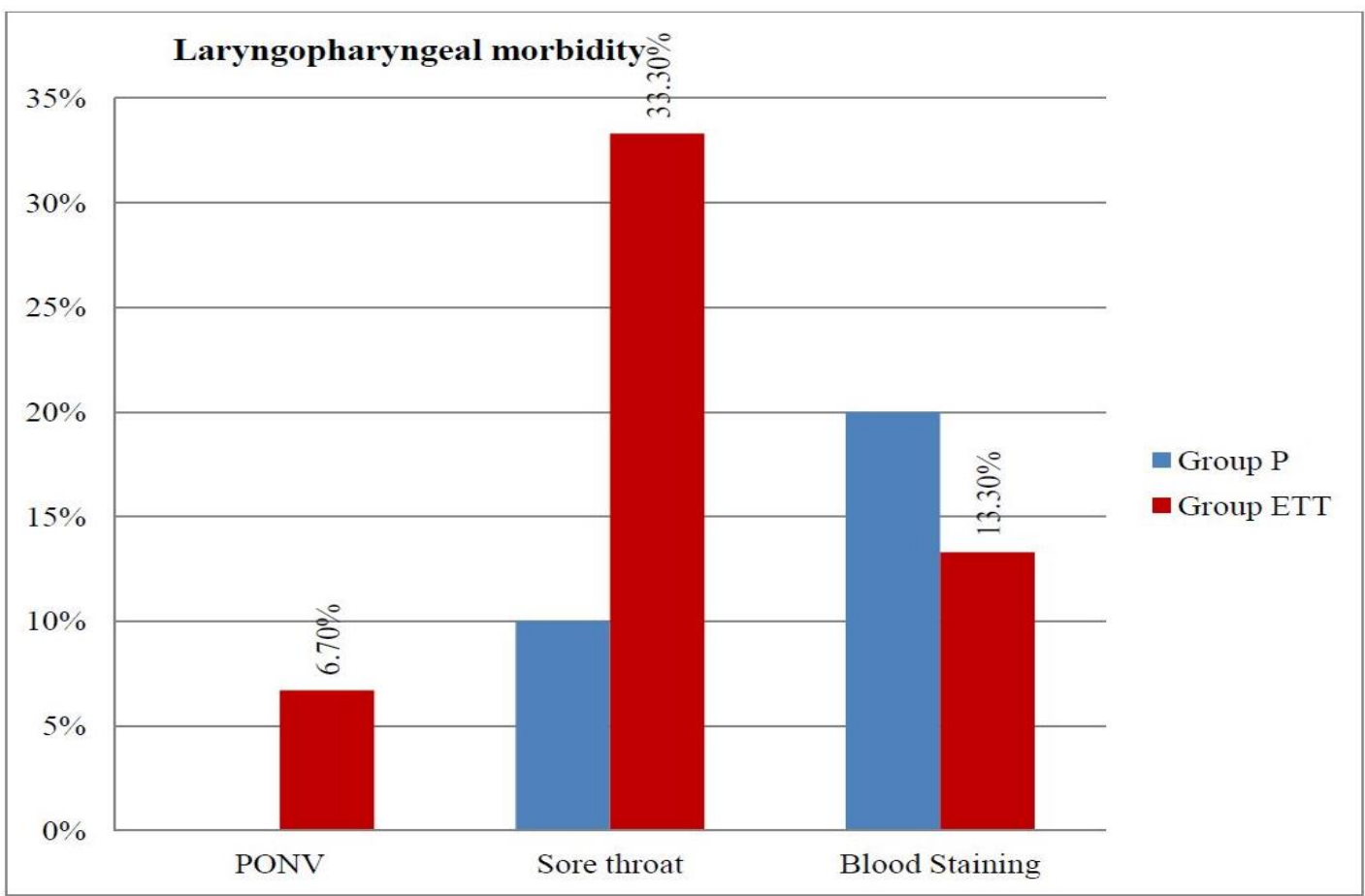

Fig. 1: Bar diagram showing laryngopharyngeal morbidity comparison between two groups 


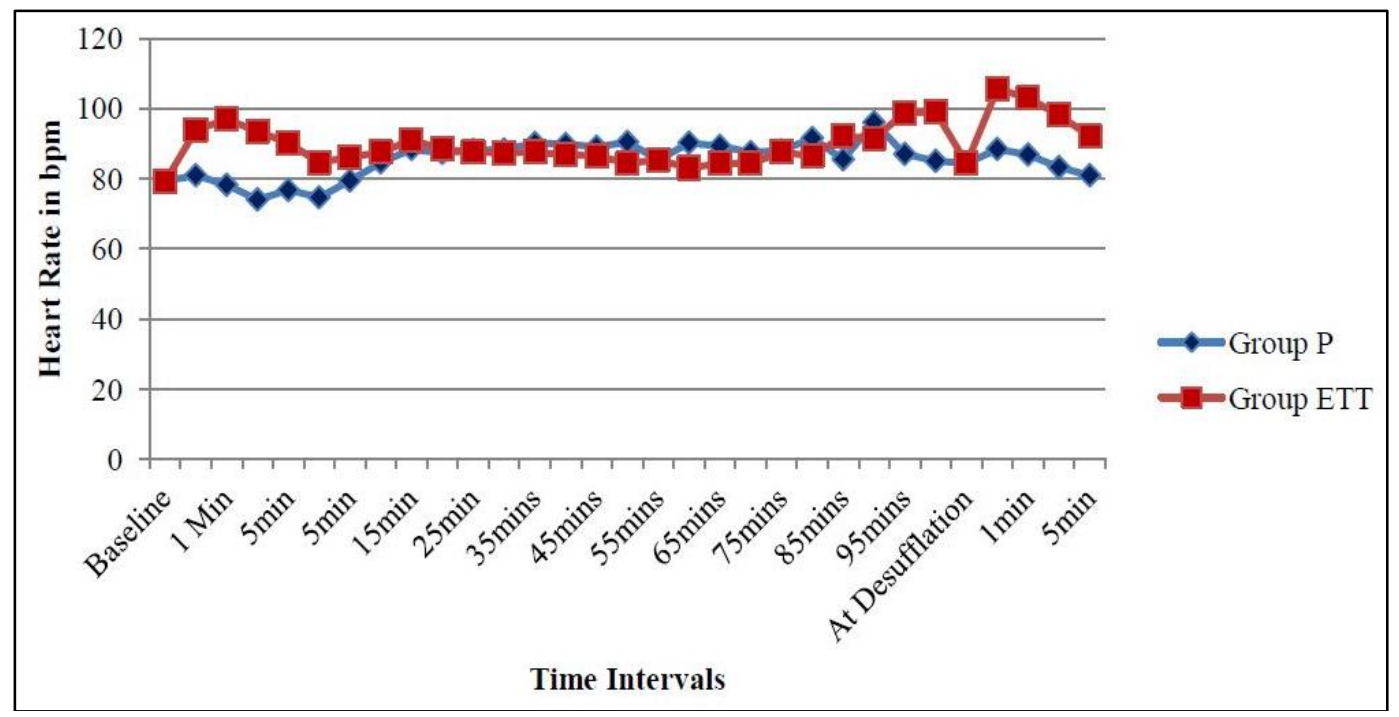

Fig. 2: Line diagram showing mean heart rate comparison between two groups at different time intervals

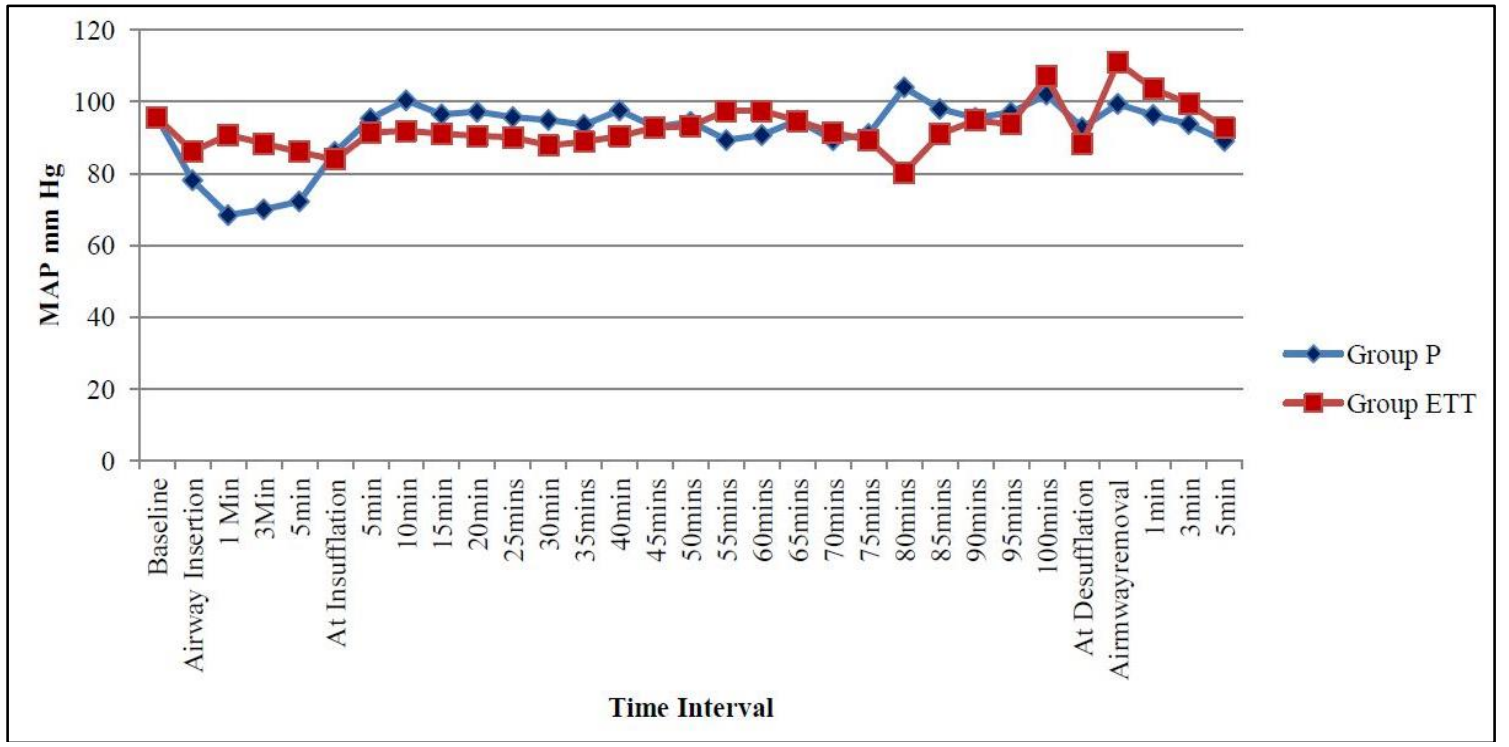

Fig. 3: Line diagram showing MAP comparison between two groups at different time intervals

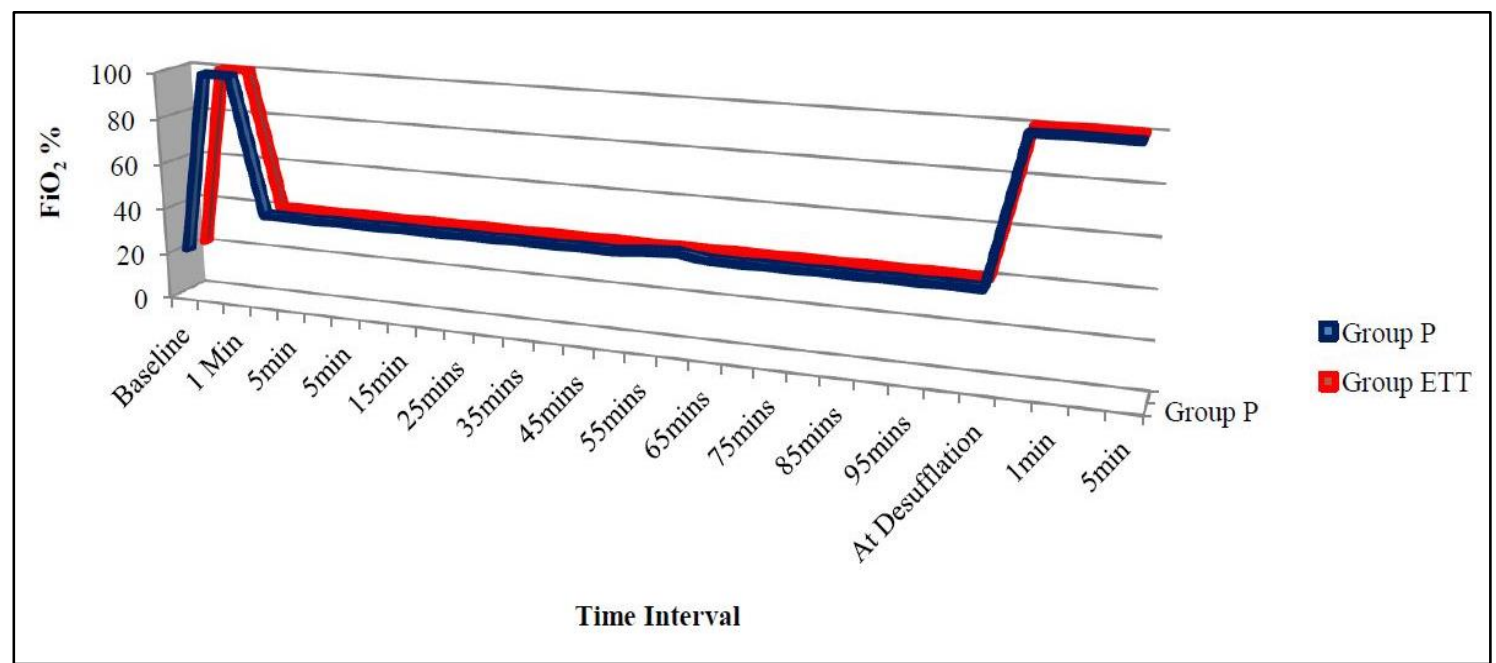

Fig. 4: Line diagram showing Mean FiO2 comparison between two groups at different time intervals 


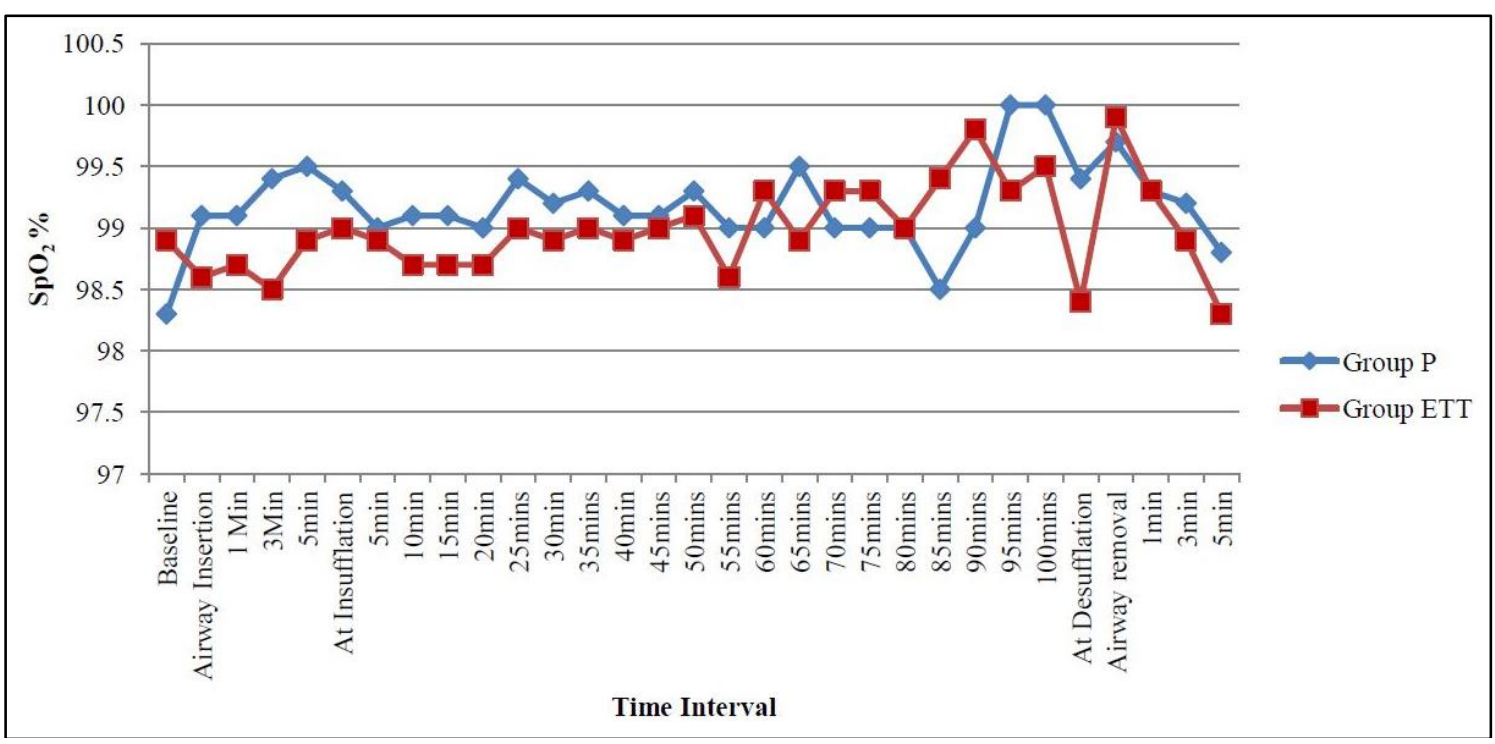

Fig. 5: Line diagram showing Mean SpO2 comparison between two groups at different time intervals

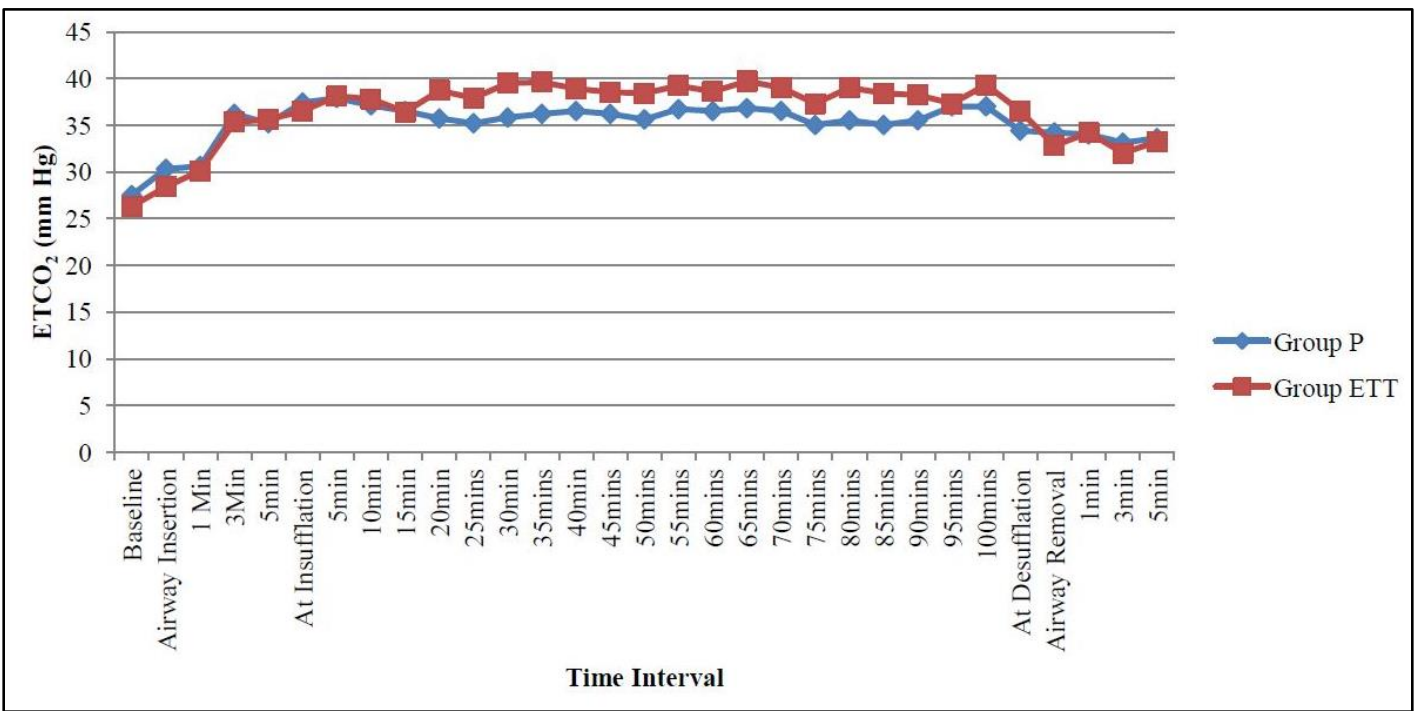

Fig. 6: Line diagram showing Mean ETCO2 comparison between two groups at different time intervals 


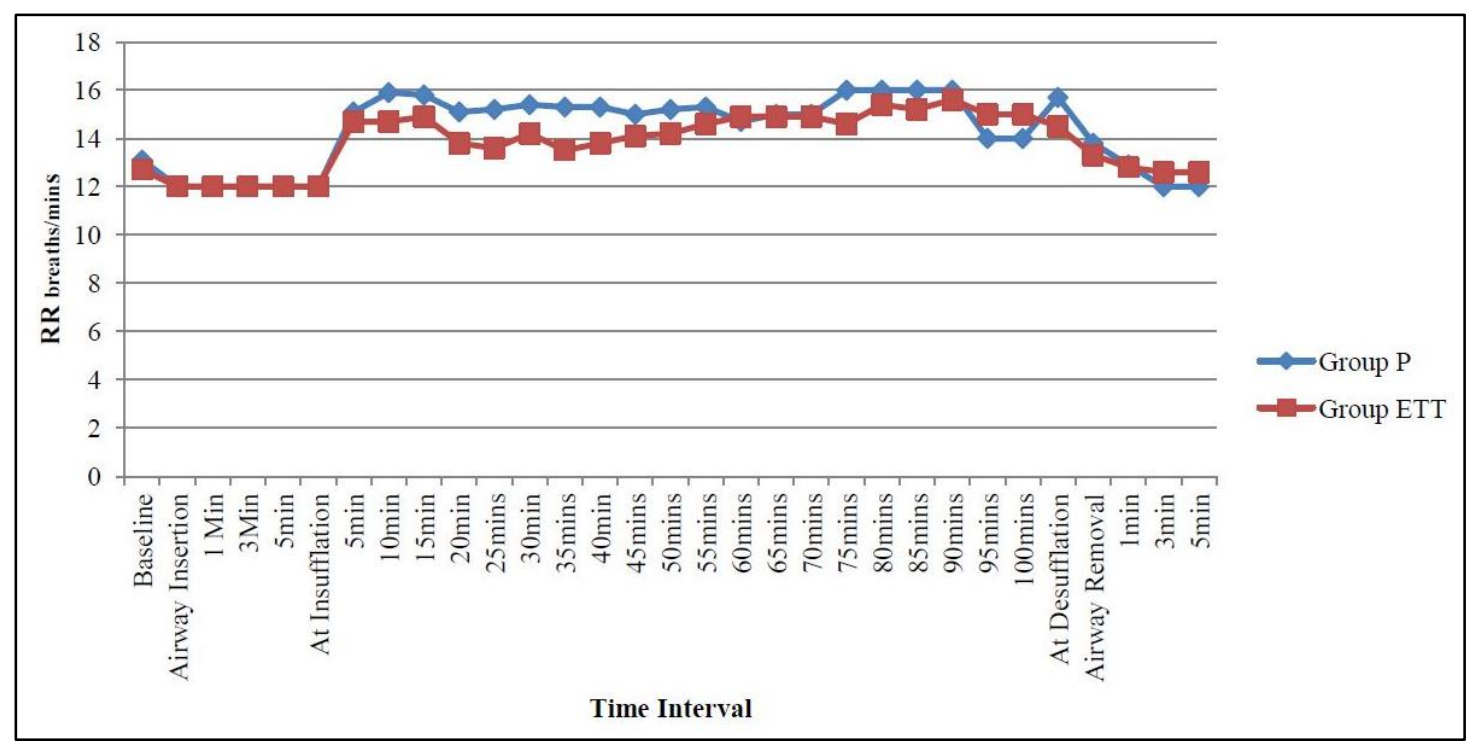

Fig. 7: Line diagram showing Mean RR comparison between two groups at different time intervals

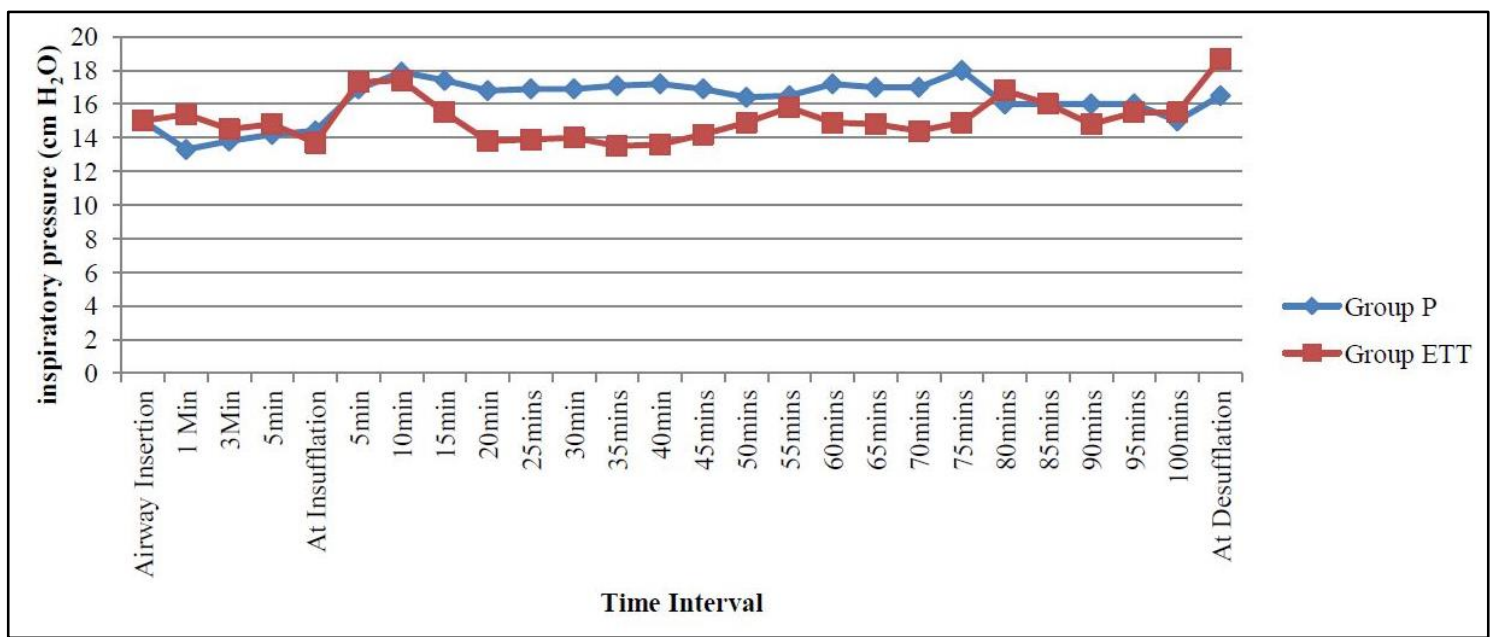

Fig. 8: Line diagram showing Mean Inspiratory Pressure comparison between two groups at different time intervals

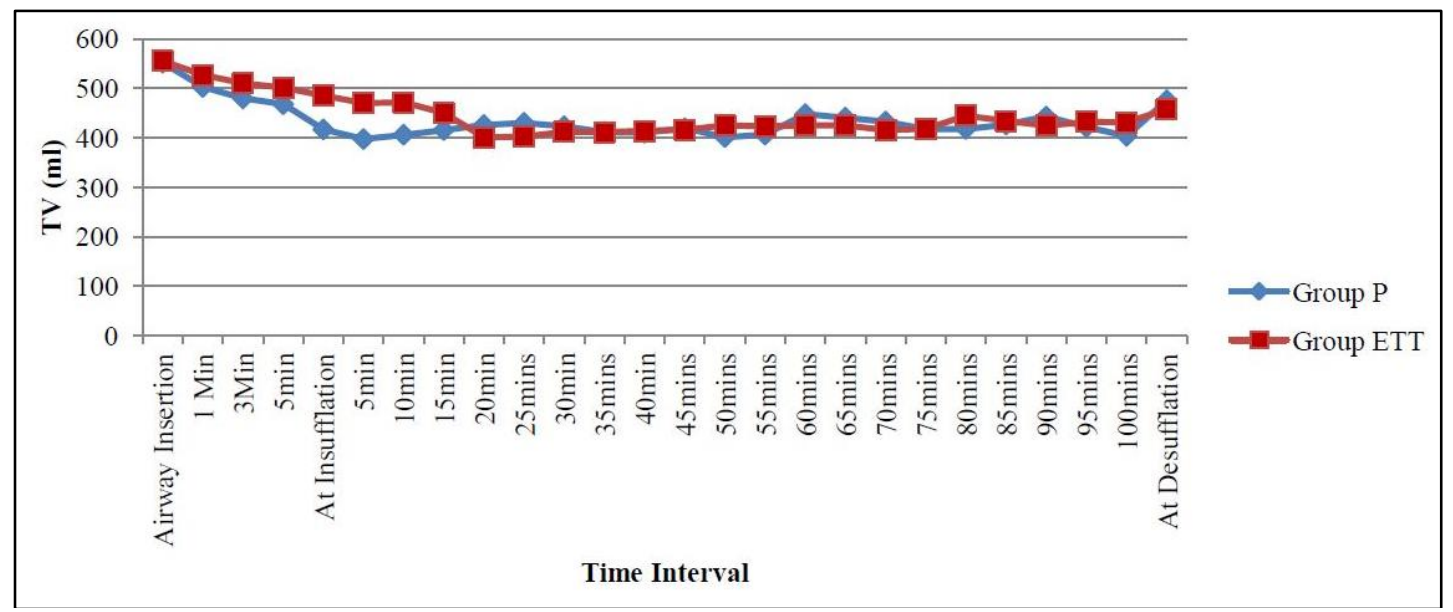

Fig. 9: Line diagram showing Mean TV comparison between two groups at different time intervals 


\section{Discussion}

Proseal laryngeal mask airway is a relatively newer airway device. We compared PLMA with ETT for oxygenation and ventilation in patients undergoing elective laparoscopic surgery. It is a known fact that the increased intra-abdominal pressures associated with pneumoperitoneum require higher airway pressures to be generated for adequate pulmonary ventilation. There have been a few studies on the use of PLMA as an airway device in laparoscopic surgeries. ${ }^{5-7}$

The primary objective of this study was to compare proseal LMA with endotracheal tube as an airway device for patients undergoing elective laparoscopic surgeries under general anaesthesia. Adequacy of oxygenation was assessed in terms of the $\mathrm{FiO}_{2}$ changes required to maintain $\mathrm{SpO}_{2}>95 \%$ and the adequacy of ventilation was assessed by the changes required in inspiratory pressure and/or respiratory rate to maintain an end-tidal carbon dioxide $\left(\mathrm{EtCO}_{2}\right)$ between $35-45 \mathrm{~mm}$ Hg.

After initiation of pneumoperitoneum, rate of transfer of $\mathrm{CO}_{2}$ into blood depends on various factors including duration of pneumoperitoneum. ${ }^{8}$ Also pneumoperitoneum is associated with splinting of the diaphragm which results in lesser pulmonary compliance and hence the need for higher pressure for similar tidal volumes prior to pneumoperitoneum. In our study, perioperative oxygenation and ventilation parameters were well maintained in both groups. $\mathrm{FiO}_{2}$ changes to maintain $\mathrm{SPO}_{2}>95 \%$ was not needed in both groups at all the intervals of follow-up. Maltby et $\mathrm{al}^{9}$ and Sharma et $\mathrm{al}^{5}$ who did a similar study found no statistical difference in the $\mathrm{SpO}_{2}$ and $\mathrm{EtCO}_{2}$ between the two groups during pneumoperitoneum.

We noted a significant difference in the mean inspiratory pressure between the two groups especially during the period of pneumoperitoneum. Patients with PLMA required higher pressure and increased respiratory rate during pneumoperitoneum in comparison to the ETT group. Sharma and colleagues ${ }^{5}$ also noted similar findings in their study. $\mathrm{EtCO}_{2}$ was increased to more than $50 \mathrm{mmHg}$ in three of their patients. They attributed this to downfolding of epiglottis. Brimacombe and his colleagues found the incidence of such downfolding of epiglottis to be about $31-66 \%{ }^{10}$ Therefore the higher resistance associated with PLMA may be the reason for the requirement of slightly higher pressure supports and respiratory rates. Even though this difference was significant, it was within acceptable range, and the $\mathrm{EtCO}_{2}$ remained well within the specified targets.

There was minimum haemodynamic stress response with PLMA when compared with endotracheal intubation. In our study we observed that the mean HR and MAP was higher in ETT group in comparison to the PLMA group at airway insertion and up to about 5 minutes after insertion and similarly at removal and upto 5 minutes after removal of airway device. Laryngoscopy and endotracheal intubation causes autonomic stimulation resulting in hypertension and tachycardia. ${ }^{11,12}$ Since laryngoscopy is not needed for insertion of PLMA, the autonomic stimulation and ensuing catecholamine release is decreased comparatively. ${ }^{13}$ In patients with cardiac illness, raised ICP and hypertension, this can make a difference in morbidity.

Although ETT was easier to insert with higher success rate in the first attempt $(100 \%)$ in comparison to PLMA $(76.7 \%)$, the mean time taken was comparable (ETT $32.2 \mathrm{~s}$ and PLMA 30.6s) and not statistically significant. This first time success rate at insertion of PLMA was similar to the study done by J.M Belena et al. ${ }^{14}$ In our study this may be attributed to lesser experience and familiarity in the use of PLMA as compared to ETT.

Average time taken to insert Ryle's tube nasally in intubated patients (74.8s) was significantly higher than time taken to insert it through the PLMA (21.2s). Also first time success rate for the same was higher in PLMA (90\%) than with ETT (70\%). In PLMA group, OPLP of $31 \mathrm{mmHg}$ was noted. Neither leak nor increase in peak airway pressure beyond OPLP was noted during surgery. OPLP observed in our study was similar to the values noted in earlier studies. ${ }^{5,9,14-16}$ There have been many studies comparing PLMA with other SADs like LMA-Supreme and Igel ${ }^{17,18-20}$ They have found that both of these devices are suitable alternatives in laparoscopic surgeries, but PLMA has higher OPLP compared to other SADs. Therefore PLMA may be the most suited device for laparoscopic surgeries among SADs.

The mean intra-abdominal pressure during pneumoperitoneum in PLMA group and in the intubated group was not very different, values being 13 and $12 \mathrm{mmHg}$ respectively.

In both groups, there were no instances of aspiration. But in PLMA group, the incidence of sore throat $(10 \%)$ was much lower than in the ETT group $(33 \%)$. Similar findings regarding sore throat were noted by Higgins et $\mathrm{al}^{21}$ and Shroff et $\mathrm{al}^{7}$. The pressures exerted on the mucosal surfaces are much less with PLMA and this can explain the difference in incidence of sore throat. ${ }^{22}$

Jose M Belenaetal published a review article on the role of LMA in laparoscopic cholecystectomy and suggested following inclusion guidelines regarding use of LMA in laparoscopic cholecystectomy which include: elective surgeries, ASA 1-3, BMI $<30 \mathrm{~kg} / \mathrm{m}^{2}$, intra-abdominal pressure of less than $13 \mathrm{mmHg}$, LMAs with drain channel to be used always. ${ }^{23}$

Limitations of the study include a. The outcome is applicable to patients with normal respiratory physiology. The acceptable increase in airway pressure and respiratory rate in this study group may be different with adverse impact in patients with altered respiratory mechanics. b. This study is an observational study. 
Hence there is no randomisation or blinding involved which could possibly have resulted in some form of bias and hence impacting the validity of results.

To conclude, PLMA is a good alternative airway device to ETT in laparoscopic surgeries. Oxygenation and ventilation parameters were well maintained with very minimal complications with PLMA. Also, the autonomic stimulation with PLMA was much lesser which can be of benefit in patients with ischemic heart disease, raised intracranial pressure etc. It provided equally effective pulmonary ventilation even though the airway pressures required were relatively high without any increase in gastric distension, aspiration and regurgitation.

\section{Conflict of Interest: None}

\section{References}

1. Peppard SB, Dickens JH. Laryngeal injury following short-term intubation. Ann Otol Rhinol Laryngol. 1983;92:327-330.

2. Gal TJ. Airway management. In: Miller RD, editor. Textbook of anaesthesia. $6^{\text {th }}$ ed. Philadelphia: Elsevier, 2005. Pp. 1617-1652

3. Jayashree S. Laryngeal mask airway and its varients. Indian J Anaesthe. 2005;49:275-280.

4. Namita S, Aditya K, Abhijeet m, Amrita G, Uma S. The comparison of proseal laryngeal mask airway and endotracheal tube in patients undergoing laparoscopic surgeries under general anaesthesia. Indian J Anaesth. 2011;55(2):129-134.

5. Sharma B, Sood J, Sahai C, Kumar VP. Efficacy and safety performance of proseal laryngeal mask airway in laparoscopic surgery: Experience in 1000 cases. Indian J Anaesth. 2008;52:288-296.

6. Misra MN, Ramamurthy B. The Pro-seal LMA and the tracheal tube: A comparison of events at insertion of the airway device. Internet J Anaesthesiol. 2008; Vol.16.

7. Shroff P, Surekha K. Randomized comparative study between the Proseal laryngeal mask airway and the endotracheal tube for laparoscopic surgery. Internet $J$ Anaesthesiol. 2006; Vol. 11.

8. Piper J. Physiological equilibria of gas cavities in the body. In: Fenn WO, Rahn M, editors. Handbook of Physiology. Section 3: Respiration. Washington DC: American Physiological Society; 1965. pp.1205-20.

9. Maltby JR. The LMA Proseal is an effective alternative to tracheal intubation for laparoscopic cholecystectomy. Can J Anaesth. 2002;49:857-862.

10. Brimacombe J. The Proseal laryngeal mask airway: an easier and safer approach to tracheal tube/laryngeal mask exchange. Anaesthesia. 2003;97;1192-1194.

11. Evans NR, Gardner SV, James MF, King JA, Roux P, Bennett $P$. The Proseal laryngeal mask: results of a descripitive trial with experience of 300 cases. $\mathrm{Br} \mathrm{J}$ Anaesth. 2002;88:534-539.

12. Fujii Y, Tanaka H, Toyooha H. Circulatory responses to laryngeal mask airway insertion or tracheal intubation in normotensive and hypertensive patients. Can J Anaesth. 1997;44:1082-1086.

13. Lamb K, James MF, Janicki PK. Laryngeal mask airway for intraocular surgery, effects on intraocular pressure and stress responses. Br J Anaesth. 1992;69:143-147.

14. J.M. Belena. Use of second generation supra-glottic airway devices during lapa-roscopic cholecystectomy: a prospective, randomized comparison of LMA Proseal ${ }^{\mathrm{TM}}$, LMA Supreme ${ }^{\mathrm{TM}}$ and i-gelT ${ }^{\mathrm{M}}$. Acta Anaesthesiol Belg, 2016:67:121-128

15. Sharma B, Sahai C, Bhattacharya A, Kumar VP, Sood J. Proseal laryngeal mask airway: A study of 100 consecutive cases of laparoscopic surgery. Indian $J$ Anaesth. 2003;47:467-472.

16. Brain AI, Verghese C, Strube PJ. The LMA `Proseal` - a laryngeal mask with an oesophageal vent. Br J Anaesth. 2000;84:650-654.

17. McItem Turkay, Hacer Sebnem, Sibel Oba. Can Supreme LMA be an alternative to endotracheal intubation in laparoscopic surgery? Braz J Anaesthesiol. 2014;64(1):66-70.

18. Belena JM, Nunez M, Anta D. Comparison of laryngeal mask airway supreme and LMA proseal with respect to oropharyngeal leak pressure during laparoscopic cholecystectomy: a randomized controlled trial. Eur J Anaesthesiol. 2013;30:119-123.

19. Anand LK, Goel N, Singh M. Comparison of the Supreme and Proseal LMA in patients undergoing laparoscopic cholecystectomy: A randomized controlled trial. Acta Anaesthesiol Taiwan. 2016;54(2):44-50.

20. Mishra SK, Sivaraman B, Balachander H, Nagappa. Effect of pneumoperitoneum and Trendelenburg position on oropharyngeal seal pressure of Igel and LMA Proseal in laparoscopic gynecologic surgery: A randomized controlled trial. Anesth Essays Res. 2015;9(3):291-455.

21. Higgins PP, Chung F, Mezei G. Postoperative sore throat after ambulatory surgery. Br J Anaesth. 2002;88:582-584.

22. Rabey PG, Murphy PJ, Langton JA, Barker P, Rowbotham DJ. The effect of the laryngeal mask airway on lower oesophageal sphincter pressure in patients during general anaesthesia. Br J Anaesth. 1992;69:621630.

23. Jose M Belena, Ernesto Josne Ochoa, Monica Menez, Carlos Gilsanz. Role of laryngeal mask airway in laparoscopic cholecystectomy. World J Gastro Intest Surg. 2015;7(11):319-325.

How to cite this article: Devendran D, Jain K. M, Upadhyaya V. K.S. Comparison of oxygenation and ventilation parameters between proseal laryngeal mask airway and endotracheal tube in patients undergoing laparoscopic surgeries: a prospective observational study. Indian J Clin Anaesth. 2018;5(4):529-537. 